\title{
AUSENCIA DE BIOTOXINA ACIDO DOMOICO EN LOS PANTANOS DE VILLA CHORRILLOS, PERÚ
}

\section{ABSENCE OF DOMOIC ACID BIOTOXIN AT VILLA WETLANDS, CHORRILLOS, PERU}

\author{
Pedro P. Álvarez F. ${ }^{1}$ y Carlos A. Santiago Ch. ${ }^{2}$
}

\begin{abstract}
Resumen
En el verano del 2003 se produjo un fenómeno calificado como «marea roja» en un espejo de agua de los Pantanos de Villa de Lima, Perú. Con el objetivo de explicar el posible incremento de muerte de aves acuáticas, se analizó el agua en busca de la biotoxina ácido domoico. Se obtuvo una muestra de agua de color rojo y se analizó para ácido domoico por cromatografía líquida de alta performance (HPLC). El equipo empleado fue un cromatógrafo Shimadzu modelo LC-10A, con inyector automático y detector UV con arreglo de diodos. Finalmente no logró aislarse ácido domoico en la muestra obtenida. Se hallaron microorganismos del género Euglena con cromatóforos rojos. Se concluye que la ausencia de ácido domoico en los Pantanos de Villa Chorrillos puede explicar que no continuaran los reportes de muertes de aves acuáticas, que fue admitido como un hecho circunstancial. La coloración roja del espejo de agua se podría explicar por la presencia de microorganismos del género Euglena. Se discuten los resultados, los efectos de la eutrofización, la comparación con sucesos similares, la presencia de ácido domoico en humedales, como la importancia del monitoreo permanente.
\end{abstract}

Palabras clave: ácido domoico; Euglena; humedal; marea roja; Villa

\begin{abstract}
The present work was carried out with the aim of determining if in the open water, during prevailing conditions of a phenomenon designated as «red tide» in the 2003 summer, at Villa Wetlands, Chorrillos, Lima, Peru, domoic acid, a biotoxin, could explain the possible increase in waterfowl deaths. A red color water sample was analyzed for domoic acid by high performance liquid chromatography (HPLC), using a Shimatzu LC-10 A equipment with an automatic injector and UV detection with a photodiode array detector. No domoic acid was found in the sample. Many microorganisms of the Euglena genus were found with red cromatophores. In conclusion, the lack of domoic acid in the Villa Wetlands, Chorrillos may explain why waterfowl deaths did not continue to rise and were considered only as a circumstantial event. The red coloration in the open water was due to the presence of microorganisms of the Euglena genus. The effects of eutrophication, similar findings in other places and the importance of continuous monitoring are discussed.
\end{abstract}

Key words: domoic acid; Euglena; red tide; Villa; Wetland

\section{Introducción}

El ácido domoico es una biotoxina producida por algas unicelulares, que ocasionalmente pueden contaminar a organismos marinos que se alimentan de ellas por filtración. Estos a su vez pueden intoxicar a lobos marinos y aves marinas que se alimentan de ellos. Los alimentos marinos contaminados pueden además intoxicar al humano ocasionando problemas de salud pública, ocasionar pérdidas enormes en la vida salvaje marina y en la economía de cualquier país exportador de alimentos marinos (Gilbert \& Pitcher, 2001).

La biotoxina aparece temporalmente, en particular en la estación de verano y suele estar ligada a la presencia de «mareas rojas» en el mar, fenómenos naturales producidos por la floración de algas unicelulares que la biosintetizan. Tales algas unicelulares son protistas eucarióticas que abundan en aguas frías como el Atlántico, siendo escasas en las aguas cálidas, perteneciendo mayoritariamente al género Pseudonitzschia (Pan et al., 2001). La biotoxina está apareciendo paulatinamente en casi todos los mares del mundo, atribuido a varios factores entre ellos la eutrofización. En América del Sur se le ha encontrado en mares de Argentina, Brasil, Uruguay y Chile, aunque no en el mar peruano.

En 1987 en Canadá fue reportada una epidemia humana por la ingestión de mejillones (Mytilus edulis) contaminados con ácido domoico (Debonnel et al., 1990). Los intoxicados presentaron a las pocas $h$ de la ingesta, náuseas, dolor abdominal y diarreas; en algunos casos también pérdida permanente de los mecanismos de la memoria reciente, atribuida a necrosis selectiva de las neuronas del hipocampo 
(Teitelbaum, 1990; Lefebvre, 2002). En 1991, 1998 y 2000, el ácido domoico produjo epidemias severas en animales marinos, con muerte de aves (Sierra et al., 1997; Kizer, 1994) y mamíferos (Lefebvre et al., 1999; Gulland, 2002) por centenares. Tales sucesos se observaron en las playas de la Bahía Monterrey, Estado de California, USA, así como en el Cabo de San Lucas, península de Baja California, México. Desde entonces se ha intensificado el monitoreo por vía satélite en diversos mares y se analizan el agua y productos marinos para garantizar su inocuidad y prevenir la intoxicación por la ingesta de alimentos contaminados con la biotoxina.

De otro lado, se han descrito «mareas rojas» en humedales, lagos y aguas dulceacuícolas en diversos lugares del mundo. Se ha encontrado la biotoxina en un posible humedal, producida por una nueva diatomea del género Nitzschia (Kotaki et al., 2000). Los humedales son extensiones de marismas, pantanos o superficies cubiertas de aguas, sean éstas de régimen natural o artificial, permanentes o temporales, estancadas o corrientes, dulces, salobres o saladas, incluidas las extensiones de agua marina cuya profundidad en marea baja no es mayor de seis metros. La idea común es que son áreas en que la capa freática aflora, quedando cubiertos por agua poco profunda (Maltchik \& Callisto, 2004).

En el verano del 2003, entre Enero y Marzo, los diarios, noticieros radiales y televisivos de Lima, Perú, informaron que un sector o espejo de agua de los Pantanos de Villa del distrito de Chorrillos, había adquirido una coloración roja similar a una «marea roja», con un posible incremento en la muerte de aves acuáticas que llegaban de paso o habitaban en tales humedales.

La Zona Reservada de los Pantanos de Villa, son humedales localizados en el distrito de Chorrillos a 30 $\mathrm{km}$ al sur de la ciudad de Lima, con alrededor de 260 ha. Está reconocida desde 1997 por la Convención Ramsar, Tratado Intergubernamental de conservación de humedales, por su importancia internacional como hábitat de aves acuáticas que dependen ecológicamente de esos humedales. Su contenido de agua se debe al afloramiento de la capa freática. La evaporación aumenta la presencia de sales, haciéndola de tipo salobre, que persiste por su proximidad al mar. La nubosidad es habitual y la precipitación pluvial irrelevante. La temperatura en el verano se encuentra entre $28^{\circ} \mathrm{C}$ a $30^{\circ} \mathrm{C}$ y la humedad relativa entre $82 \%$ y 93\%. Existen muchas especies de aves acuáticas migratorias y residentes, peces, algas unicelulares y protozoarios del género Euglena (Guillén et al., 2003).

El objeto del presente trabajo fue determinar si en el sector de los humedales de Villa Chorrillos, donde se estaba produciendo el cambio de color de sus aguas, se hallaba la biotoxina ácido domoico, que pudiera explicar el posible incremento en la muerte de aves acuáticas.

\section{Materiales y métodos}

\section{Equipo y reactivos}

El análisis para la biotoxina se realizó por cromatografía líquida de alta perfomance (HPLC) empleando un cromatógrafo Shimadzu modelo LC$10 \mathrm{~A}$, con inyector automático y detector UV con arreglo de diodos (longitud de onda variable), que incluyó bomba con sistema de degasificación, horno para la termostatización de la columna, sistema de registro y procesado de datos cromatográficos en computadora con software original. Se usó una columna cromatográfica Supelco de acero inoxidable C-18 para fase reversa, de 150 x $4.6 \mathrm{~mm}$ de diámetro interno y $5 \mu \mathrm{m}$ de diámetro de partícula. El ensayo cromatográfico se realizó en un sistema isocrático, con flujo de $1.25 \mathrm{~mL} \cdot \mathrm{min}^{-1}$, volumen de inyección de 10 $\mu \mathrm{L}$, detección UV en $242 \mathrm{~nm}$, temperatura del horno en $40^{\circ} \mathrm{C}$ y tiempo de corrida de $15 \mathrm{~min}$.

Los reactivos metanol grado HPLC, acetonitrilo grado HPLC y ácido trifluoracético (grado espectrofotométrico), se adquirieron en Merck ${ }^{\circledR}$ en Lima. Se utilizó agua tridestilada. La fase móvil consistió de una solución de acetonitrilo: agua: ácido trifluoracético (10: 89.9: 0.1; v/v). Para la observación se consideró el tiempo de retención de un estándar de ácido domoico.

Obtención de la muestra

A mediados de Febrero de 2003 hacia las 12 del mediodía, fue ubicado el espejo de agua en un área del extremo noreste de los Pantanos de Villa Chorrillos, entre los $12^{\circ} 11^{\prime} 42^{\prime \prime}$ - $-12^{\circ} 13^{\prime} 18^{\prime \prime}$ 'S y $76^{\circ} 58^{\prime} 42^{\prime \prime}$ $76^{\circ} 59^{\prime} 42^{\prime \prime}, \mathrm{W}$, como el único que presentaba coloración roja y que se distinguía desde alrededor de $50 \mathrm{~m}$ de distancia. A continuación, empleando guantes de látex, fueron sumergidos lentamente dos frascos limpios transparentes de vidrio borosilicato de aproximadamente un L cada uno, a fin de obtener las muestras desde un nivel entre tres a cinco $\mathrm{cm}$ de la superficie del agua, siendo finalmente cerrados y etiquetados. En una búsqueda cuidadosa, no se advirtieron aves muertas en los alrededores.

En el laboratorio, se confirmó el color rojo translúcido del líquido colectado. El contenido de los frascos fue trasvasado a dos vasos de precipitado y puestos en refrigeración a $4^{\circ} \mathrm{C}$ en oscuridad, a fin de ser trabajados para el ensayo de ácido domoico en las siguientes 24 a 48 h. Antes de ser puestos en refrigeración se tomaron varias gotas de la muestra para su examen directo en preparados simples, aplicando una gota sobre un portaobjetos y cubriéndola con una laminilla de 18 x $18 \mathrm{~mm}$ para la búsqueda del posible organismo biológico involucrado en el color rojizo. Para la observación se empleó un microscopio Olympus binocular compuesto con luz incorporada (Carl Zeiss), usando los objetivos 4x, 10x y 20x así como el ocular 10x. 
Preparación de la muestra

Cerca de $800 \mathrm{~mL}$ de la muestra fue llevada al rotavapor para su evaporación hasta obtener alrededor de $20 \mathrm{~mL}$. Luego, se tomó $4 \mathrm{~mL}$ del concentrado, se agregó $16 \mathrm{~mL}$ de metanol al 50\% y se homogenizó por 10 min en el vortex. Después se centrifugó a 5000 rpm por $15 \mathrm{~min}$. Se formaron dos capas, una que acumulaba la coloración roja y otra el sobrenadante transparente. Cinco $5 \mathrm{~mL}$ del sobrenadante fue pasado a través de filtros de membrana de $0.45 \mathrm{~mm}$. Se continuó con un método validado para ácido domoico (Pérez, 2002).

Al día siguiente de concluida la primera muestra, se realizó el mismo procedimiento con la cantidad restante de la muestra.

\section{Resultados}

La observación microscópica de las gotas de las muestras encontró microorganismos correspondientes al género Euglena, los cuales presentaban coloración roja. El objetivo del estudio fue determinar la presencia del ácido domoico, si se hallaba se continuaba con la determinación del género Euglena. La clave dicotómica taxonómica empleada estuvo basada en un número limitado de caracteres microscópicos que incluyeron la morfología, el flagelo y el estigma (Kudo, 1969). De otro lado, el género Euglena en los pantanos de Villa ha sido ampliamente descrito desde tiempo atrás (Sarmiento \& Morales, 1998; Guillén et al., 2003).

En las muestras corridas en el equipo HPLC, en ambas ocasiones, no se halló ácido domoico.

Discusión

Con la contaminación eutrófica marina, las «mareas rojas» parecen ser más frecuentes y aún más peligrosas por el florecimiento de algas unicelulares del género Pseudonitzschia que pueden sintetizar el ácido domoico. Esta biotoxina no se encuentra siempre ligada a las «mareas rojas», porque se le ha aislado en mares sin coloración. La posibilidad de que una «marea roja» en humedales o lagos pueda ser debida a el florecimiento de diatomeas del género Pseudonitzschia no ha sido descrita aún. En un humedal en Vietnam (Kotaki et al., 2000) ha sido aislada una diatomea productora de ácido domoico que es la Nitzschia navis-varingica, aislada en aguas salobres de una laguna camaronícola. También se le ha encontrado en aguas salobres en áreas tropicales y temperadas de Japón y Manila, produciendo ácido domoico (Kotaki et al., 2004) e incluso ácidos isodomoico A y B (Kotaki et al., 2005).

A diferencia de Pseudo-Nitzschia, que pertenece a la misma familia y se desarrollan en climas fríos, $N$. navis-varingica es de clima tropical y subtropical. Hasta antes de dicho hallazgo, no se había establecido claramente que el ácido domoico pudiera detectarse en aguas dulces o salobres, propias de los humedales. Ello, sumado a la información inicial en los noticieros en Lima sobre un posible incremento de la muerte de aves, lo cual no se demostró posteriormente, orientó la búsqueda de dicha biotoxina. No llegamos a visualizar alguna ave sin vida y no se volvió a escuchar sobre muerte de aves.

En la observación directa microscópica el microorganismo predominante fue del género Euglena que presentaba cromatóforos de color rojo. La presencia de Euglena sp. ya ha sido descrita en los humedales de Villa y de otras regiones del mundo, por lo que puede ser considerado un hallazgo común. La "marea roja" en el espejo de agua de los Pantanos, podría explicarse por la presencia de Euglena sp. con pigmentos de ese color, que ha ocurrido en otros humedales como el Lago Manasabal de Cachemira, India (Khan \& Bhat, 2000), en el que la invasión de la Euglena pedunculata Gojdics causó áreas extensas de agua de color rojo. La «marea roja» del lago Manasabal de la India, se hizo recurrente a varios lagos de Cachemira (Khan, 2000), incluyendo el lago Dal y el lago Wular, ambos por E. pedunculata. Para explicar el fenómeno inicialmente se manejó la hipótesis de la precipitación ácida por el petróleo quemado durante la guerra del Golfo, aunque finalmente se admitió la hipótesis de la eutrofización por el influjo de aguas residuales no tratadas, al hierro y altas temperaturas ambientales. En otro caso, las mareas rojas por Euglena sp. en los humedales de Barak Valley, Assam, India, se encontró que eran inducidas por las altas concentraciones de $\mathrm{NO}_{3}, \mathrm{Fe}$ y $\mathrm{Mg}$ entre otros elementos traza, acumulados por los organismos de la marea, la cual colapsó cuando las concentraciones de esos elementos disminuyeron en el agua, encontrando además que la marea no fue tóxica al ser utilizada como alimento por los peces (Duttagupta et al., 2004). Esta ausencia de toxicidad puede descartar que la "marea roja” en los Pantanos de Villa hubiera sido responsable de la muerte de aves, observadas al inicio del fenómeno.

El Servicio de Parques de Lima informó que el color rojizo del espejo de agua del Pantano de Villa procedía de la excesiva reproducción de Euglena clorophoenicea, cuya característica principal es la presencia de hematocromos (cromatóforos de color rojo) en el interior de su cuerpo, que producía ese color cuando la temperatura ambiental alcanzaba los $30^{\circ} \mathrm{C}$, lo que coincide con lo descrito en el Orden Euglenoidida (Kudo, 1969). El hallazgo del género Euglena como responsable de la «marea roja» coincidió con el nuestro, la causa básica de su presentación podría ser también la eutrofización. En general, el que este fenómeno se presente por temporadas anuales debe ser atribuido a que los niveles de contaminación o eutrofización no disminuyen sino permanecen o aumentan.

Fue importante demostrar que los análisis no aislaron la biotoxina en las muestras tomadas, ello se explica porque no se ha descrito que los 
microorganismos del género Euglena sean capaces de sintetizar ácido domoico y al parecer no existen algas productoras de la biotoxina en los Pantanos de Villa. Considerando el descubrimiento de nuevos microorganismos que pueden sintetizar el ácido domoico y la eutrofización cada vez mayor a nivel mundial y regional, como otros factores no descritos en la síntesis de dicha biotoxina, siempre será útil el monitoreo continuo e intervenir cuando se sospeche de una eventualidad de esta naturaleza, para evitar las repercusiones en la salud pública y en la economía. Máxime si ha sido encontrado ácido domoico, aunque en muy bajos niveles no tóxicos, en mariscos cosechados del lago Aveiro en Portugal en el año 2001 (Vale \& Sampayo, 2002).

Se concluye señalando que en el verano del 2003, con la aparición de la «marea roja», posiblemente producida por la floración del género Euglena, no se encontró la biotoxina ácido domoico en el espejo de agua analizado de los Pantanos de Villa.

\section{Literatura citada}

Debonnel G., Weiss M. \& de Montigny C. 1990. Neurotoxic effect of domoic acid: mediation by kainate receptor electrophysiological studies in the rat. Can. Dis. Wkly. Rep. 16 Suppl 1E: 59-68.

Duttagupta S., Gupta S. \& Gupta A. 2004. Euglenoid blooms in the floodplain wetlands of Barak Valley, Assam, North eastern India. J. Environ. Biol. 25(3): 36973.

Glibert P. \& Pitcher G. 2001. Global Ecology and Oceanography of Harmful Algal Blooms (GEOHAB); science plan. An International Programme sponsored by the Scientific Committee on Oceanic Research (SCOR) and the Intergovernmental Oceanographic Commission (IOC). UNESCO document.

Guillén G., Morales E. \& Severino R. 2003. Adiciones a la fauna de protozoarios de los Pantanos de Villa, Lima, Perú. Rev. per. biol. 10(2): 175-182.

Gulland F.M., Haulena M., Fauquier D., Langlois G., Lander M.E., Zabka T. \& Duerr R. 2002. Domoic acid toxicity in Californian sea lions (Zalophus californianus): clinical signs, treatment and survival. Vet. Rec. 150(15): 475-80.

Khan M.A. \& Bhat G.H. 2000. Biological invasión and «red water» phenomenon in Lake Manasabal of Kashmir Valley, India. Polln. Res. 19(1): 113-117.

Khan M.A. 2000. Anthropogenic Eutrophication and Red Tide Outbreak in Lacustrine Systems of the Kashmir Himalaya. Acta hydrochim. hydrobiol. 28(2): 95-101.

Kizer K.W. 1994. Domoic acid poisoning. West. J. Med. 161(1): 59-60.

Kotaki Y., Koike K., Yoshida M., Thuoc C.V., Huyen N.T.M., Hoy N.C., Fukuyo Y. \& Kodama M. 2000. Domoic acid production in Nitzschia sp. (bacillariophyceae) isolated from a shrimp-culture pond in Do Son, Vietnam. J. Phycol. 36: 1057-1060.
Kotaki Y., Lundholm N., Onodera H., Kobayashi K.F.F., Bajarias F.A., Furio E., Iwataki M., Fukuyo Y. \& Kodama M. 2004. Wide distribution of Nitzschia navisvaringica, a new domoic acid-producing benthic diatom found in Vietnam. Fish. Sci. 70(1): 28-32.

Kotaki Y., Furio E.F., Satake M., Lundholm N., Katayama T., Koike K., Fulgueras V.P., Bajarias F.A., Takata Y., Kobayashi K., Sato S., Fukuyo Y. \& Kodama M. 2005. Production of isodomoic acids $\mathrm{A}$ and $\mathrm{B}$ as major toxin components of a pennate diatom Nitzschia navisvaringica. Toxicon. 46(8): 946-53.

Kudo R. 1969. Protozoologia. Compañía Editorial Continental (CECSA). México.

Lefebvre K.A., Powell C.L., Busman M., Doucette G.J., Moeller P.D., Silver J.B., Miller P.E., Hughes M.P., Singaram S., Silver M.W. \& Tjeerdema R.S. 1999. Deteccion of domoic acid in northern anchovies and Californian sea lions associated with an unusual mortality event. Nat. Toxins. 7(3): 85-92.

Lefebvre K.A., Silver M.W., Coale S.L. \& Tjeerdema R.S. 2002. Domoic acid in planktivorous fish in relation to toxic Pseudo-nitzschia cell densities. Mar. Biol. 140: 625-31.

Maltchik L. \& Callisto M. 2004. The use of rapid assessment approach to discuss ecological theories in wetland systems, Southern Brazil. Interciencia 29(4): 219-23.

Pan Y., Parsons M.L., Busman M., Moeller P.D.R., Dortch Q., Powell C.L. \& Doucette G.J. 2001. Pseudo-nitzschia sp. cf. pseudodelicatissima -a confirmed producer of domoic acid from the northern Gulf of Mexico. Mar. Ecol. Prog. Ser. 220: 83-92.

Pérez C.R. 2002. Validación de la metodología para la determinación de ácido domoico (biotoxina ASP) en moluscos bivalvos por cromatografía líquida de alto rendimiento (HPLC). Rev. per. med. Exp. salud publica. 19:142-8.

Sarmiento L. \& Morales E. 1998. Protozoarios, Turbelarios y Nematodos de los Pantanos de Villa, Lima.

Perú: Su Importancia en el Ecosistema. En: Cano, A.; R. Young (Eds.) 1998 Pantanos de Villa. Biología y Conservación. Museo de Historia Natural - UNMSM. : 41-54.

Sierra Beltran A., Palafox-Uribe M., Grajales-Montiel J., Cruz-Villacorta A. \& Ochoa J.L. 1997. Sea bird mortality at Cabo San Lucas, Mexico: evidence that toxic diatom blooms are spreading. Toxicon. 35(3): 44753.

Teitelbaum J.S., Zatorre R.J., Carpenter S., Gendron D., Evans A.C., Gjedde A. \& Cashman N.R. 1990. Neurologic sequelae of domoic acid intoxication due to the ingestion of contaminated mussels. N. Eng.l J. Med. 322(25): 1781-7.

Vale P. \& de M Sampayo M.A. 2002. First confirmation of human diarrhoeic poisonings by okadaic acid esters after ingestion of razor clams (Solen marginatus) and green crabs (Carcinus maenas) in Aveiro lagoon, Portugal and detection of okadaic acid esters in phytoplankton. Toxicon. 40(7): 989-96.

\footnotetext{
${ }^{1}$ Centro Nacional de Control de Calidad, Instituto Nacional de Salud. Av. Defensores del Morro (ex-Huaylas) 2268. Chorrillos, Lima. PERU. E-mail: taquifilaxia@yahoo.com

2Centro Nacional de Alimentación y Nutrición, Instituto Nacional de Salud, Lima, Perú.
} 